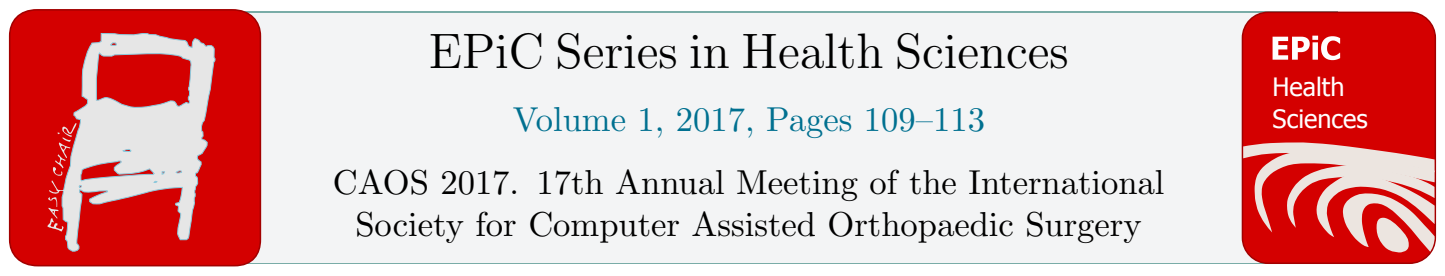

\title{
Biomechanical models of the hip - A validation study based on 10 CT-datasets
}

\author{
Jörg Eschweiler ${ }^{1,3^{*}}$, Malte Asseln ${ }^{1}$, Philipp Damm², Maximilian C.M. \\ Fischer $^{1}$, Klaus Radermacher ${ }^{1}$ \\ ${ }^{1}$ Chair of Medical Engineering, Helmholtz-Institute for Biomedical Engineering, RWTH Aachen \\ University, Germany \\ ${ }^{2}$ Julius Wolff Institut, Charité - Universitaetsmedizin Berlin, Berlin, Germany \\ ${ }^{3}$ Department for Orthopedic Surgery, University Hospital Aachen, Germany \\ * eschweiler@hia.rwth-aachen.de
}

\begin{abstract}
Consideration of the pre- and post-operative magnitude of the hip joint force $\mathrm{R}$ and its orientation $\Theta$ is of major importance for satisfactory long-term results in total hip arthroplasty. $\mathrm{R}$ and $\Theta$ can be computed by using biomechanical models with adapted geometrical/ anthropometrical parameters taken from clinical X-ray images. The objective of this study was to evaluate the models of Pauwels and Debrunner based on digital reconstructed-radiographs (central projection) from $10 \mathrm{CT}$-datasets of patients treated with telemetric hip-implants by a comparison to corresponding in-vivo measurements.

$\mathrm{R}$ and $\Theta$ were computed for 10 patients with patient-specific geometric/anthropometric parameters. The model adaption was based on 28 anatomical landmarks. The root-mean-square-error of $\mathrm{R}$ is smaller for Debrunner (0.59/vs./0.66), and for $\Theta$ it is smaller for Pauwels' (4.47/vs./7.78).

Mathematical models provide potentially valuable information regarding hip joint mechanics. Regarding R, in all of the 10 patients the predictions of Pauwels' model are consistently higher than the in-vivo measurements. Debrunner computed R in 8 cases higher and in 2 cases lower than the corresponding in-vivo forces. Pauwels' and Debrunner showed similar tendencies: in 8 cases an overestimation of $\mathrm{R}$ and in 2 cases contrary results. Regarding $\Theta$ we found that in 5 cases the predictions of Pauwels' are consistently higher than the in-vivo measurements and also contrary to Debrunner.

As previous studies showed, an unambiguous identification of most landmarks in a 2D X-ray image is difficult. The impact of the pelvic tilt on the computational result was not considered in our study. Further investigation of this aspect is part of our ongoing work.
\end{abstract}




\section{Introduction}

Biomechanical models of the hip have the potential to predict the resultant joint force $\mathrm{R}$ and its orientation $\Theta$ (Zhang et al., 2015). Knowledge of hip joint mechanics is important for a variety of reasons e.g. understanding the function of normal and diseased joint situation for surgical planning, designing better implants, rehabilitation regimens, and evaluating the effects of treatment (Brand et al., 1994).

Especially the consideration of the pre- and post-operative magnitude of $\mathrm{R}$ and its orientation $\Theta$ is of major importance in order to achieve satisfactory long-term results in total hip arthroplasty (THA). $\mathrm{R}$ and $\Theta$ can be computed by using patient-specific biomechanical models with adapted geometrical and anthropometrical parameters taken from standardized acquired clinical X-ray images. In the context of developing a planning module for computer-assisted THA, the models of Pauwels, Debrunner, Blumentritt and Iglič were investigated in earlier studies (Eschweiler et al., 2012). However, it has been shown that the models of Blumentritt and Iglič yielded contradictory results in comparison to others as well as compared to the in-vivo measurements (Eschweiler et al., 2012). Blumentritt's model computed R too high, and Iglič's revealed a lack of patient-specific adaption.

Therefore, the objective of this study was to evaluate the mathematical models of Pauwels, and Debrunner based on digital reconstructed radiographs (DRRs) from $10 \mathrm{CT}$-datasets of patients treated with telemetric hip implants (OrthoLoad, (Bergmann et al., 2015)) by a comparison to corresponding in-vivo measurements.

\section{Materials and Methods}

The magnitude of $\mathrm{R}$ and its orientation $\Theta$ was computed for 10 patients (mean age 57 years, 6 left and 4 right implants, 8 males and 2 females, mean height $174 \mathrm{~cm} \pm$ SD $5.9 \mathrm{~cm}$, mean weight $88.7 \mathrm{~kg}$ $\pm \mathrm{SD} 12.4 \mathrm{~kg}$ ) with patient-specific geometric and anthropometric parameters acquired from DRR's of corresponding CT-datasets.

The geometrical model adaption was based on 28 anatomical landmarks. The model predictions were directly compared to their corresponding in-vivo measurements obtained from instrumented hip implants of the open access OrthoLoad-database.

The DRRs were created by using a central projection. The coordinate transformation from CT scanner to the camera coordinate system was done by the following equations (Equation 1):

Equation 1: Coordinate transformation

$X_{C, i}=X_{i}$

$Y_{C, i}=Y_{i}+\frac{F D A}{M A G} * 100$

$Z_{C, i}=Z_{i}-\left(Z_{C, P S}+0,05\right)$

with a magnification (MAG) of $110 \%$, a focus detector distance (FDA) of $1.15 \mathrm{~m}$ and the Zcoordinate of the pubic symphysis according to (Debrunner, 1975). 


\section{Results}

Examples of the results for the computation of $\mathrm{R}$ are shown in Figure 1, the results of $\Theta$ are shown in Figure 2, and furthermore, they are summarized in Table 1.

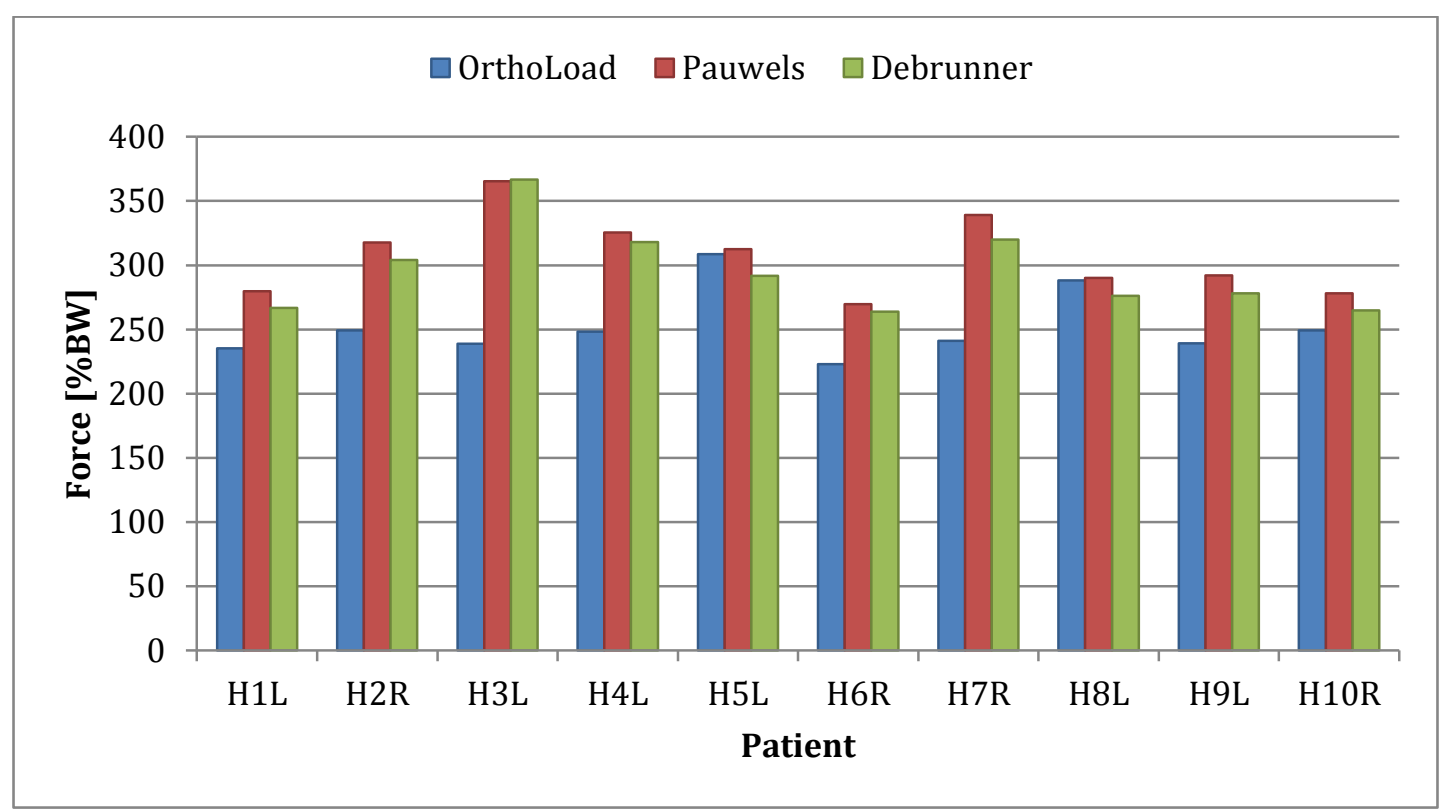

Figure 1: Results for the deviation of the hip joint resultant force $R$ in case of central projection for creating DRRs.

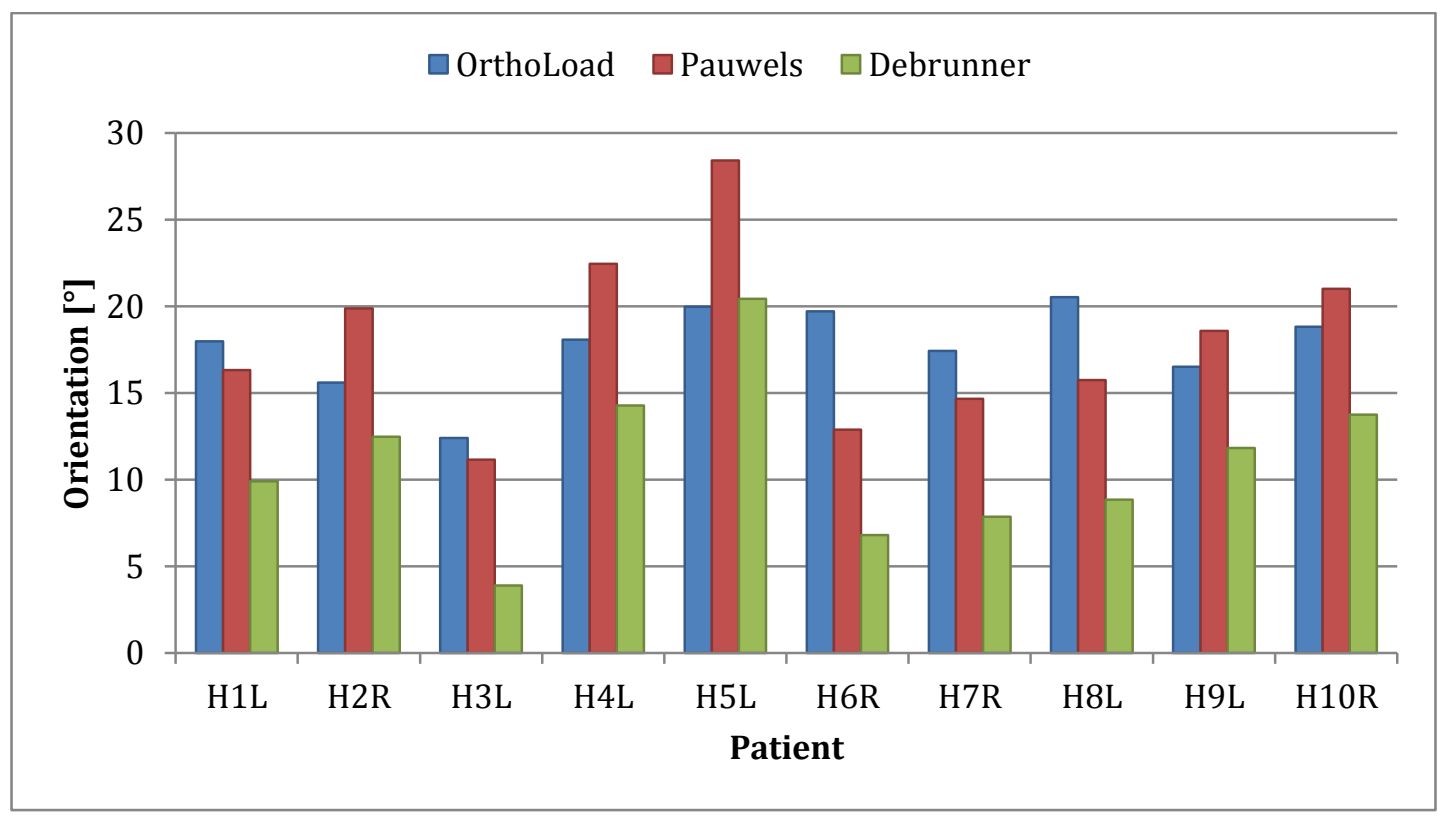

Figure 2: Results for the orientation of $R$ in case of central projection for creating DRRs. 
Table 1: Results for the central projection

\begin{tabular}{lcc}
\hline Model & $\begin{array}{c}\text { RMSE } \\
\text { amplitude }\end{array}$ & $\begin{array}{c}\text { RMSE } \\
\text { orientation }\end{array}$ \\
Pauwels & R $[\% \mathbf{B W}])$ & $\left(\boldsymbol{\Theta}\left[^{\circ}\right]\right)$ \\
Debrunner & 0.66 & 4.47 \\
\hline
\end{tabular}

Table 1 shows that the root-mean-square error (RMSE) of R is slightly smaller for Debrunner's model, and the RMSE of $\Theta$ is smaller for Pauwels'.

\section{Discussion}

Mathematical models, capable to calculate joint forces and its orientation, provide potentially valuable and patient-specific information regarding hip joint (bio)mechanics for THA planning. We evaluated the mathematical models of Pauwels, and Debrunner. Therefore, we used DRRs from 10 CT-datasets of patients treated with telemetric hip implants and compared the computation results to the corresponding in-vivo measurements.

Regarding the resultant force R, we found that in all of the 10 patients the predictions of Pauwels' model are consistently higher than the in-vivo measurements. Debrunner's model computed R in 8 cases higher and in 2 (H5L, H8L) cases lower than the corresponding in-vivo forces, however, both the predictions and measurements exhibit reasonable internal consistency. Furthermore, Pauwels' and Debrunner's models showed similar tendencies: in 8 cases an overestimation of $\mathrm{R}$ was observed, and in 2 cases (H5L, H8L) the results of both model calculations were contrary. In comparison to the invivo results the differences were not negligible. The reasons for the discrepancy in some patients could be the simplification of a 3D problem to a 2D mathematical model, which could be more relevant in some patients than in others. Clinically, a prediction of a higher $\mathrm{R}$ would be more critical, hence potentially leading e.g. to a recommendation of a bigger offset.

Regarding $\Theta$ we found that in 5 cases (H2R, H4L, H5L, H9L, H10R) the predictions of Pauwels' model are consistently higher than the in-vivo measurements and also contrary to Debrunner's model. Here, the results were too low in all cases. Clinically, a smaller $\Theta$ would stand for a higher risk of critical edge loading and dislocation, hence potentially leading e.g. to a recommendation of a smaller inclination (which in turn would have an impact on the resulting range of motion (ROM) or risk of impingement, respectively).

There are limitations coming along with our study. As previous studies showed (Dell'Anna et al., 2010), an unambiguous identification of most landmarks in an 2D X-ray image is difficult, which could be an explanation for differences from the in-vivo results. The impact of the pelvic tilt on the computational result was not considered in our study (CT supine position vs. standing X-rays as assumed for the models of Debrunner and Pauwels). Further investigation of this aspect is part of our ongoing work.

In conclusion, the preliminary results of our study motivate the consideration of biomechanical models in the context of CAOS and therapy planning, respectively. 


\section{References}

Bergmann et al., 2015. OrthoLoad Database. http://www.orthoload.com/. Julius Wolff Institute Charité Universitaetsmedizin Berlin.

Brand, R.A., Pedersen, D.R., Davy, D.T., Kotzar, G.M., Heiple, K.G., Goldberg, V.M., 1994. Comparison of hip force calculations and measurements in the same patient. The Journal of arthroplasty $9,45-51$.

Debrunner, H.U., 1975. Biomechanics of the hip joint. I. A new model for the calculation of the forces in the hip joint. Z Orthop Ihre Grenzgeb 113, 377-388.

Dell'Anna, J., Fieten, L., Eschweiler, J., Maus, U., Radermacher, K., 2010. Sensitivity analysis of a biomechanical score fpr cup positioning in total hip arthroplasty. Proceedings of the 10th Annual Meeting of CAOS-International, Paris, June 16-19th, 364-366.

Eschweiler, J., Fieten, L., Dell'Anna, J., Kabir, K., Gravius, S., Tingart, M., Radermacher, K., 2012. Application and evaluation of biomechanical models and scores for the planning of total hip arthroplasty. Proceedings of the Institution of Mechanical Engineers, Part H: Journal of Engineering in Medicine 226, 955-967.

Zhang, X., Chen, Z., Wang, L., Yang, W., Li, D., Jin, Z., 2015. Prediction of hip joint load and translation using musculoskeletal modelling with force-dependent kinematics and experimental validation. Proceedings of the Institution of Mechanical Engineers, Part H: Journal of Engineering in Medicine 229, 477-490. 